\title{
Hamiltonian Formulation of Heat Conduction: Integral Transform Method Approach
}

\author{
V. Bertola ${ }^{\mathrm{a}, *}$ and E. Cafaro ${ }^{\mathrm{b}}$ \\ ${ }^{a}$ School of Engineering and Electronics, University of Edinburgh, Edinburgh EH9 3JL, United Kingdom and ${ }^{b}$ Diparti- \\ mento di Energetica, Politecnico di Torino, 10129 Torino, Italy
}

\begin{abstract}
The Hamiltonian formulation of heat conduction is derived using the integral transform method approach, in comparison with the conventional Hamiltonian formulation in the physical space of the variables temperature and heat flux. An analogy with the relativistic motion of a particle is briefly discussed.
\end{abstract}

\section{INTRODUCTION}

The mathematical description of physical systems makes often use of the Hamiltonian formalism, which from its original application to derive the so-called Hamilton's equations of motion in classical mechanics quickly spread on many other physical phenomena, ranging from statistical physics to quantum mechanics [1]. Although the Hamiltonian formulation of a problem of mathematical physics in general does not simplify the search for a solution, very often it provides a better insight of physical phenomena and reveals analogies which may remain hidden when using other formulations.

The description of heat conduction in a material of constant thermophysical properties using the Hamiltonian formalism was first proposed by Magyari and Keller [2] in the case of one-dimensional, boundary value problems, for which the energy conservation equation and Fourier's law are:

$$
\begin{aligned}
& \rho c \frac{\partial T(x, t)}{\partial t}+\frac{\partial J(x, t)}{\partial x}=0 \\
& J(x, t)=-k \frac{\partial T(x, t)}{\partial x}
\end{aligned}
$$

where $T(x, t)$ is temperature, $J(x, t)$ the heat flux, $\rho, c$, and $k$ are respectively the density, the heat capacity and the thermal conductivity of the material.

Separation of variables allows one to write the temperature and the heat flux in the form $T(x, t)=A(x) \exp (-t / \tau)$ and $J(x, t)=-B(x) \exp (-t / \tau)$, where $\tau$ is the separation constant, so that Eq. (1) can be re-written as:

$$
\begin{aligned}
& \dot{B}(x)=-\frac{\rho c}{\tau} A(x) \\
& \dot{A}(x)=\frac{1}{k} B(x)
\end{aligned}
$$

This can be immediately converted to the Hamiltonian form $\dot{A}(x)=\partial H / \partial B$ and $\dot{B}(x)=-\partial H / \partial A$, where $H$ is the Hamiltonian function defined as:

*Address correspondence to this author at the The University of Edinburgh, School of Engineering and Electronics, King's Buildings, Mayfield Road, Edinburgh EH9 3JL, United Kingdom; Tel: +44-131-6508697; Fax: +44131-6506551; E-mail:V.Bertola@ed.ac.uk or mailto:V.Bertola@ed.ac.uk
$H=\frac{\rho c}{2 \tau} A^{2}+\frac{1}{2 k} B^{2}$

In this approach, a problem of heat conduction becomes equivalent to the motion of a fictitious particle of mass $k$, position $A$, momentum $B$, and total energy $H$, where time is represented by the spatial coordinate. Besides the invariance of the quantity defined in Eq. (3), an immediate practical consequence of the Hamiltonian formulation is the interchangeability of the temperature and heat flux variables, which allows one to transform a problem in the variable $T$ (temperature) with Neumann boundary conditions into a problem in the variable $J$ (heat flux) with Dirichlet boundary conditions. Another benefit is the generalized use of the socalled propagator method, where the solution of a heat conduction problem can be obtained by solving a simple matrix equation [2], which has significant computational advantages in comparison with conventional methods.

The aim of this note is to show how a general Hamiltonian formulation of heat conduction can be obtained straightforward by using an integral transform method approach. In fact, in the Laplace space problems are always solved using the propagator method, and thus the properties of the Hamiltonian function (including its invariance) are strictly related to those of the transformation matrix.

\section{ANALYSIS}

The integral transform method is an elegant and powerful technique to solve various problems of mathematical physics, which has a number of advantages in comparison with classical methods when they are applied to the solution of partial differential equations [3]. In the case of heat conduction problems [4], the common practice is replacing temperature with its Laplace transform, $\widetilde{T}(s)=\int_{0}^{+\infty} T(t) e^{-s t} d t$, so that the heat equation in the direction normal to isothermal surfaces, $v$, becomes:

$\rho \operatorname{cs} \widetilde{T}=k \frac{d^{2} \widetilde{T}}{d v^{2}}$

This second-order equation is equivalent to the following system of first-order equations: 


$$
\left\{\begin{array}{l}
\frac{d \widetilde{T}}{d v}=-\frac{\widetilde{J}}{k} \\
\frac{d \widetilde{J}}{d v}=-\rho c s \widetilde{T}
\end{array}\right.
$$

which can be re-written in matrix form as:

$$
\frac{d \mathbf{X}}{d v}=\mathbf{A} \cdot \mathbf{X}
$$

where $\mathbf{X}=\left(\begin{array}{l}\widetilde{T} \\ \widetilde{J}\end{array}\right)$ and $\mathbf{A}=\left(\begin{array}{cc}0 & -1 / k \\ -\rho c s & 0\end{array}\right)$ is the matrix of the material properties.

Equation (6) represents the evolution (propagation) in space of the transformed quantities along a path that is locally perpendicular to isothermal surfaces, and its solution is given by:

$$
\mathbf{X}=\exp (\mathbf{A} v) \mathbf{X}_{0}
$$

where the vector rotation with respect to the initial condition $\mathbf{X}_{0}$ is determined by the matrix:

$\exp (A v)=\sum_{n=0}^{\infty} \frac{(\mathbf{A} \boldsymbol{v})^{n}}{n !}=$

$$
=\left(\begin{array}{cc}
\operatorname{Cosh}\left(v \sqrt{\frac{s}{\alpha}}\right) & -\frac{\operatorname{Sinh}\left(v \sqrt{\frac{s}{\alpha}}\right)}{\sqrt{\rho c k s}} \\
-\sqrt{\rho c k s} \operatorname{Sinh}\left(v \sqrt{\frac{s}{\alpha}}\right) & \operatorname{Cosh}\left(v \sqrt{\frac{s}{\alpha}}\right)
\end{array}\right)
$$

and $\alpha$ is the thermal diffusivity.

The problem must be obviously completed with appropriate boundary conditions. For example, given an infinite slab of thickness $\mathrm{L}$ that separates two fluids with periodically fluctuating temperatures $T_{f, 0}=a_{0} \cos \left(\omega_{0} t\right) \quad$ and $T_{f, L}=a_{L} \cos \left(\omega_{L} t\right)$, one finds the following Neumann boundary conditions:

$$
\left\{\begin{array}{l}
-k\left(\frac{\partial T}{\partial x}\right)_{0}=h_{0}\left[a_{0} \cos \left(\omega_{0} t\right)-T_{0}\right] \\
-k\left(\frac{\partial T}{\partial x}\right)_{L}=h_{L}\left[T_{L}-a_{L} \cos \left(\omega_{L} t\right)\right]
\end{array}\right.
$$

where $h_{0}$ and $h_{L}$ are the convective heat transfer coefficients between the slab and the two fluids, respectively. After Laplace transformation these boundary conditions become:

$$
\left\{\begin{array}{l}
\widetilde{J}_{0}=h_{0}\left(\frac{a_{0} s}{s^{2}+\omega_{0}^{2}}-\widetilde{T}_{0}\right) \\
\widetilde{J}_{L}=h_{L}\left(\widetilde{T}_{L}-\frac{a_{L} s}{s^{2}+\omega_{L}^{2}}\right)
\end{array}\right.
$$

Thus, the propagator vectors on the boundaries are, respectively:

$$
\mathbf{X}_{0}=\left(\begin{array}{cc}
1 & 0 \\
-h_{0} & -h_{0}
\end{array}\right) \cdot\left(\begin{array}{c}
\widetilde{T}_{0} \\
-\frac{a_{0} s}{s^{2}+\omega_{0}^{2}}
\end{array}\right)
$$

and

$$
\mathbf{X}_{L}=\left(\begin{array}{cc}
1 & 0 \\
h_{L} & h_{L}
\end{array}\right) \cdot\left(\begin{array}{c}
\widetilde{T}_{L} \\
-\frac{a_{L} s}{s^{2}+\omega_{L}^{2}}
\end{array}\right)
$$

From Eqs. (7), (8) and (11) one finds that the solution is given by:

$$
\begin{aligned}
& \left(\begin{array}{c}
\widetilde{T} \\
\widetilde{J}
\end{array}\right)=\left(\begin{array}{cc}
\operatorname{Cosh}\left(x \sqrt{\frac{s}{\alpha}}\right) & -\frac{\operatorname{Sinh}\left(x \sqrt{\frac{s}{\alpha}}\right)}{\sqrt{\rho c k s}} \\
-\sqrt{\rho c k s} \operatorname{Sinh}\left(x \sqrt{\frac{s}{\alpha}}\right) & \operatorname{Cosh}\left(x \sqrt{\frac{s}{\alpha}}\right)
\end{array}\right) . \\
& \cdot\left(\begin{array}{cc}
1 & 0 \\
-h_{0} & -h_{0}
\end{array}\right) \cdot\left(\begin{array}{c}
\widetilde{T}_{0} \\
-\frac{a_{0} s}{s^{2}+\omega_{0}^{2}}
\end{array}\right)
\end{aligned}
$$

where $\widetilde{T}_{0}$ is unknown, and must be determined using the second boundary condition, Eq. (12):

$$
\begin{aligned}
& \left(\begin{array}{cc}
1 & 0 \\
h_{L} & h_{L}
\end{array}\right) \cdot\left(\begin{array}{c}
\widetilde{T}_{L} \\
-\frac{a_{L} s}{s^{2}+\omega_{L}^{2}}
\end{array}\right)= \\
& =\left(\begin{array}{cc}
\operatorname{Cosh}\left(x \sqrt{\frac{s}{\alpha}}\right) & -\frac{\operatorname{Sinh}\left(x \sqrt{\frac{s}{\alpha}}\right)}{\sqrt{\rho c k s}} \\
-\sqrt{\rho c k s} \operatorname{Sinh}\left(x \sqrt{\frac{s}{\alpha}}\right) & \operatorname{Cosh}\left(x \sqrt{\frac{s}{\alpha}}\right)
\end{array}\right) . \\
& \cdot\left(\begin{array}{cc}
1 & 0 \\
-h_{0} & -h_{0}
\end{array}\right) \cdot\left(\begin{array}{c}
\widetilde{T}_{0} \\
-\frac{a_{0} s}{s^{2}+\omega_{0}^{2}}
\end{array}\right)
\end{aligned}
$$

Equation (7) shows that the solution of heat conduction problems by the propagator method is possible by using the integral transform method: therefore the application of the propagator method to the solution of heat conduction problems cannot be considered a consequence of the Hamiltonian formulation [2].

If the Laplace transform of temperature is rescaled by introducing the new variable $\widetilde{\Phi}=\widetilde{T} \sqrt{\rho c k s}$, the spatial evolution of the variables is expressed by the following transformation: 


$$
\left(\begin{array}{c}
\widetilde{\Phi}^{\prime} \\
\widetilde{J}^{\prime}
\end{array}\right)=\left(\begin{array}{cc}
\operatorname{Cosh}\left(v \sqrt{\frac{s}{\alpha}}\right) & -\operatorname{Sinh}\left(v \sqrt{\frac{s}{\alpha}}\right) \\
-\operatorname{Sinh}\left(v \sqrt{\frac{s}{\alpha}}\right) & \operatorname{Cosh}\left(v \sqrt{\frac{s}{\alpha}}\right)
\end{array}\right)\left(\begin{array}{c}
\tilde{\Phi} \\
\widetilde{J}
\end{array}\right)
$$

Equation (15) is formally identical to a Lorentz transformation (hyperbolic rotation of coordinates) in the space defined by $\widetilde{\Phi}$ and $\widetilde{J}$ [5]. In particular, it describes the motion of a fictitious, relativistic particle along the spatial coordinate $\widetilde{J}$, according to a time variable defined as $t=\widetilde{\Phi} / c^{*}$, where $c^{*}$ is a constant representing the maximum speed attainable by the particle (i.e., the speed of light in this coordinates system). In this analogy, the dimensionless particle velocity is given by:

$\frac{u}{c^{*}}=\frac{\left[\exp \left(v \sqrt{\frac{s}{\alpha}}\right)\right]^{2}-1}{\left[\exp \left(v \sqrt{\frac{s}{\alpha}}\right)\right]^{2}+1}$

According to a well-known result of special relativity the quantity $\widetilde{J}^{2}-\widetilde{\Phi}^{2}$, which corresponds to the spacetime interval in Minkowski's space, is an invariant [6], and therefore it can be used to define a Hamiltonian function for heat conduction in Lapalce's space:

$\widetilde{H}=\frac{1}{2 k}\left(\widetilde{J}^{2}-\widetilde{\Phi}^{2}\right)$

It must be remarked that while in the traditional approach the definition of a Hamiltonian invariant relies on the separation of variables ansatz [2], in the present work the constancy of the quantity defined by Eq. (17) descends uniquely from the properties of the transformation matrix in Eq. (15).

One can easily verify that $\partial \tilde{H} / \partial v=0$, so that the Hamiltonian does not depend explicitly on the direction of the heat flux, and takes the form $\widetilde{H}=\tilde{H}(p(v), q(v))$, where $p(v)=-\widetilde{J}$ and $q(v)=\widetilde{T}$. Thus, the Laplace transforms of temperature and heat flux play the role of conjugate variables in the Hamiltonian formulation.

\section{CONCLUSIONS}

The integral transform method allows one to derive a Hamiltonian formulation of the phenomenon of heat conduction. It can be shown that in the Laplace space heat conduction is analogous to the relativistic motion of a fictitious particle moving at a smaller speed than light, and the existence of a Hamiltonian invariant is ensured by a theorem of special relativity.

An important issue arising with Hamiltonian systems, which is generally neglected when solving heat conduction problems, is their numerical integration [7]. In fact most numerical methods (including, for instance, of all Runge-Kutta methods) do not ensure the conservation of the Hamiltonian, especially in complex problems with long integration trajectories. This suggests that numerical integration methods which keep the Hamiltonian constant (i.e., symplectic methods) may be better suitable to solve heat conduction problems.

\section{REFERENCES}

[1] H. Goldstein, Classical Mechanics, Chapter 9, Reading: Addison Wesley, 1980.

[2] E. Magyari and B. Keller "Hamiltonian description of the heat conduction", Heat Mass Trans., Vol. 34, pp. 453-459, 1999.

[3] K. Yoshida and S. Ito, Functional analysis and Differential Equations, Berlin: Springer-Verlag, 1975.

[4] A.V. Luikov, Analytical Heat Diffusion Theory, New York: Academic Press, 1968.

[5] H.A. Lorentz, "Electromagnetic phenomena in a system moving with any velocity less than that of light", Proc. Acad. Sci. Amsterdam, IV, pp. 669-678, 1904.

[6] S. Weinberg, Gravitation and Cosmology: Principles and Applications of the General Theory of Relativity, Upper Saddle River: Wiley, 1972.

[7] B. Leimkuhler and S. Reich, Simulating Hamiltonian Dynamics, Cambridge: Cambridge University Press, 2005. 